\title{
Nonlocal and multiple-point fractional boundary value problem in the frame of a generalized Hilfer derivative
}

\author{
Wasfi Shatanawi ${ }^{1,2,3^{*}}$ (D), Abdellatif Boutiara ${ }^{4}$, Mohammed S. Abdo ${ }^{5} \mathbb{B}$, Mdi B. Jeelani ${ }^{6}$ and \\ Kamaleldin Abodayeh ${ }^{1}$
}

\author{
Correspondence: \\ wshatanawi@psu.edu.sa \\ ${ }^{1}$ Department of Mathematics and \\ General Sciences, Prince Sultan \\ University, Riyadh, Saudi Arabia \\ 2Department of Medical Research, \\ China Medical University Hospital, \\ China Medical University, Taichung, \\ 40402, Taiwan \\ Full list of author information is \\ available at the end of the article
}

\begin{abstract}
The aim of this manuscript is to handle the nonlocal boundary value problem for a specific kind of nonlinear fractional differential equations involving a $\xi$-Hilfer derivative. The used fractional operator is generated by the kernel of the kind $k(\vartheta, s)=\xi(\vartheta)-\xi(s)$ and the operator of differentiation $D_{\xi}=\left(\frac{1}{\xi^{\prime}(\vartheta)} \frac{d}{d \vartheta}\right)$. The existence and uniqueness of solutions are established for the considered system. Our perspective relies on the properties of the generalized Hilfer derivative and the implementation of Krasnoselskii's fixed point approach and Banach's contraction principle with respect to the Bielecki norm to obtain the uniqueness of solution on a bounded domain in a Banach space. Besides, we discuss the Ulam-Hyers stability criteria for the main fractional system. Finally, some examples are given to illustrate the viability of the main theories.
\end{abstract}

MSC: 34A08; 26A33; 34A34

Keywords: Nonlocal fractional differential equations; Generalized Hilfer fractional derivative; Existence and Ulam stability; Fixed point theorem

\section{Introduction}

Fractional calculus (FC) was introduced at the end of the seventeenth century as a branch of mathematical analysis that deals with the examinations of various possibilities to term real (or complex) number powers of the integration (and differentiation) operators. FC is the generalization of ordinary calculus concerned with operations of integration (and differentiation) of noninteger order.

Fractional differential equations (FDEs) including Caputo, Riemann-Liouville (RL), Hilfer, Hadamard, and Erdelyi-Kober fractional derivatives (FDs) have been used in different areas of technological disciplines and concentrated on by numerous mathematicians, see the books [1-5] and the references given therein. Recently, using the generalized MittagLeffler function, Atangana and Baleanu [6] suggested a new formulation of the fractional derivative with a nonlocal and nonsingular kernel. Atangana [7] introduced a new differentiation which combines fractal differentiation and fractional differentiation. Very recently,

(c) The Author(s) 2021. This article is licensed under a Creative Commons Attribution 4.0 International License, which permits use, sharing, adaptation, distribution and reproduction in any medium or format, as long as you give appropriate credit to the original author(s) and the source, provide a link to the Creative Commons licence, and indicate if changes were made. The images or other third party material in this article are included in the article's Creative Commons licence, unless indicated otherwise in a credit line to the material. If material is not included in the article's Creative Commons licence and your intended use is not permitted by statutory regulation or exceeds the permitted use, you will need to obtain permission directly from the copyright holder. To view a copy of this licence, visit http://creativecommons.org/licenses/by/4.0/. 
another methodology of FDs having a general kernel with respect to another function known as the $\psi$-Caputo and $\psi$-Hilfer operator has been introduced by Almeida [8] and Sousa and Oliveira [9], respectively, drawing on the idea of Kilbas et al. [1, 3] and Agrawal [10] who made some generalizations to fractional integrals and derivatives with respect to another function. Jarad and Abdeljawad [11] presented interesting properties of generalized operators with another function, including the generalized Laplace transform. A few specialists were as of late occupied with the advancement of $\xi$-Caputo (or $\xi$-Hilfer)-type FDEs, see [12-25] and the references therein. Impressive considerations have been provided to the investigation of the Ulam-Hyers (UH) stability of a wide range of FDEs, see [26-32].

In this regard, we investigate the existence, uniqueness, and UH stability of solutions for the following nonlocal boundary value problem:

$$
\begin{aligned}
& \mathcal{D}_{a^{+}}^{\varrho_{1}, \varrho_{2} ; \xi} z(\vartheta)=\mathcal{F}(\vartheta, z(\vartheta)), \quad \vartheta \in \mathscr{J}:=[a, b], \\
& z(a)=z_{a}+\mathfrak{p}(z), \quad z_{\xi}^{\prime}(b)=z_{b}+\sum_{i=1}^{m-2} \lambda_{i} z_{\xi}^{\prime}\left(\varkappa_{i}\right),
\end{aligned}
$$

where $z_{\xi}^{\prime}=\frac{z^{\prime}}{\xi^{\prime}}$ and $\mathcal{D}_{a^{+}}^{\varrho_{1}, \varrho_{2} ; \xi}$ is the $\xi$-Hilfer FD of order $\varrho_{1} \in(1,2)$ and type $\varrho_{2} \in[0,1], z_{a}, z_{b} \in$ $\mathscr{R}, \lambda_{i}>0, a<\varkappa_{i}<b, i=1,2, \ldots, m-2, \sum_{i=1}^{m-2} \lambda_{i} z_{\xi}^{\prime}\left(\varkappa_{i}\right):=d<1$, and $\mathcal{F}: \mathscr{J} \times \mathscr{R} \rightarrow \mathscr{R}$ and $\mathfrak{p}: C(\mathscr{J}, \mathscr{R}) \rightarrow \mathscr{R}$ are continuous.

Remark 1.1 Our results for problem (1.1)-(1.2) remain true for the following cases:

- RL-type problem for $\xi(\vartheta)=\vartheta$ and $\varrho_{2}=0$.

- Caputo-type problem for $\xi(\vartheta)=\vartheta$ and $\varrho_{2}=1$.

- $\xi$-RL-type problem for $\varrho_{2}=0$.

- $\xi$-Caputo-type problem for $\varrho_{2}=1$.

- Hilfer-type problem for $\xi(\vartheta)=\vartheta$.

- Hilfer-Hadamard-type problem for $\xi(\vartheta)=\log \vartheta$.

- Katugampola-type problem for $\xi(\vartheta)=\vartheta^{\rho}$.

Remark 1.2 Results acquired for problem (1.1)-(1.2) include the results of Zhong and Lin [33] and Asawasamrit et al. [34], in particular:

(1) For $\xi(\vartheta)=\vartheta$ and $\varrho_{2}=1$, the outcomes obtained in the present work incorporate the results of Zhong and Lin [33] for Caputo-type nonlocal and multiple-point BVP.

(2) If, in problem (1.1)-(1.2), $\xi(\vartheta)=\vartheta$ and replacing conditions $z(a)=0, z(b)=\sum_{i=1}^{m} \lambda_{i} \mathcal{I}^{\delta_{i}}\left(\varkappa_{i}\right)$ with (1.2), then our current results cover the results of Asawasamrit et al. [34] for Hilfer nonlocal BVP.

The main contribution of the current work is to determine the equivalent fractional integral equation to $\xi$-Hilfer type FDEs (1.1)-(1.2) and to explore the existence and uniqueness results. Further, we discuss the Ulam-Hyers stability result to such equations. Observe that, with the above discussions, problem (1.1)-(1.2) not just incorporates the previously specified BVPs in the literature, yet additionally nontrivially extends the status to a more comprehensive class of nonlocal BVPs, i.e., for various values of $\varrho_{2}$ and $\xi$, our considered 
problem covers the problems referenced in Remark 1.1. Consequently, problem (1.1)-(1.2) studied in this paper is novel and is the first to investigate fractional nonlocal problems of $\xi$-Hilfer type.

Here is a brief outline of the paper. Section 2 provides the definitions and preliminary facts that we will need for our forthcoming analysis. In Sect. 3, we prove the existence, uniqueness, and UH stability results for problem (1.1)-(1.2). Two examples are given in Sect. 5. This work closes with a conclusion.

\section{Preliminaries}

In this section, we give some notions regarding the fractional integrals and derivatives with respect to another function $\xi$. For more details, we refer to $[1-3,5]$.

Let $\mathscr{J}=[a, b] \subset \mathscr{R}$. Denote by $\mathcal{C}:=C(\mathscr{J}, \mathbb{R})$ the Banach space of continuous functions $\omega: \mathscr{J} \rightarrow \mathscr{R}$ with the norm

$$
\|\omega\|_{\infty}=\sup \{|\omega(\vartheta)|, \vartheta \in \mathscr{J}\} .
$$

Let $\xi, \omega \in \mathcal{C}^{n}$ such that $\xi$ is increasing and $\xi^{\prime}(\vartheta) \neq 0$ for all $\vartheta \in \mathscr{J}$.

Definition 2.1 ([9]) The $\xi$-Hilfer FD of a function $\omega$ of order $\varrho_{1} \in(n-1, n]$ and type $\varrho_{2} \in[0,1]$ is defined by

$$
\mathcal{D}_{a+}^{\varrho_{1}, \varrho_{2} ; \xi} \omega(\vartheta)=\mathcal{I}_{a+}^{\varrho_{2}\left(n-\varrho_{1}\right) ; \xi} D_{\xi}^{n} \mathcal{I}_{a+}^{\left(1-\varrho_{2}\right)\left(n-\varrho_{1}\right) ; \xi} \omega(\vartheta),
$$

where $n=\left[\varrho_{1}\right]+1, n \in \mathbb{N}$, and $D_{\xi}^{n}=\left(\frac{1}{\xi^{\prime}(\vartheta)} \frac{d}{d t}\right)^{n}$. Relation (2.1) can be written as

$$
\mathcal{D}_{a+}^{\varrho_{1}, \varrho_{2} ; \xi} \omega(\vartheta)=\mathcal{I}_{a+}^{\gamma-\varrho_{1} ; \xi R L} \mathcal{D}_{a+}^{\gamma ; \xi} \omega(\vartheta)
$$

with $\gamma=\varrho_{1}+\varrho_{2}\left(n-\varrho_{1}\right), \mathcal{I}_{a+}^{\gamma-\varrho_{1} ; \xi}(\cdot)$ and ${ }^{R L} \mathcal{D}_{a+}^{\gamma ; \xi}(\cdot)$ are the $\xi$-RL fractional integral and derivative defined by (see [1])

$$
\mathcal{I}_{a+}^{\theta ; \xi} \omega(\vartheta)=\frac{1}{\Gamma(\theta)} \int_{a}^{\vartheta} \xi^{\prime}(s)(\xi(\vartheta)-\xi(s))^{\theta-1} \omega(s) \mathrm{d} s
$$

and

$$
{ }^{R L} \mathcal{D}_{a+}^{\theta ; \xi} \omega(x)=D_{\xi}^{n} \mathcal{I}_{a+}^{n-\theta ; \xi} \omega(\vartheta),
$$

respectively. Furthermore, the $\xi$-Caputo FD is given by (see [8])

$$
{ }^{C} \mathcal{D}_{a^{+}}^{\theta ; \xi} \omega(\vartheta)=\mathcal{I}_{a+}^{n-\theta ; \xi} D_{\xi}^{n} \omega(\vartheta)
$$

where $\theta \in\left\{\gamma-\varrho_{1}, \gamma, \varrho_{1}\right\}$.

Remark 2.2 If we set $\xi(\vartheta)=\vartheta$ in relations (2.1), (2.3), (2.4), and (2.5), we obtain the classical fractional operators introduced in $[1,5,35]$. 
Lemma $2.3([1,9])$ Let $\varrho_{1}, \varrho_{2}>0$ and $\omega \in \mathcal{C}$. Then

$$
\mathcal{I}_{a+}^{\varrho_{1} ; \xi} \mathcal{I}_{a+}^{\varrho_{2} ; \xi} \omega(\vartheta)=\mathcal{I}_{a+}^{\varrho_{1}+\varrho_{2} ; \xi} \omega(\vartheta)
$$

and

$$
\mathcal{D}_{a+}^{\varrho_{1}, Q_{2} ; \xi} \mathcal{I}_{a+}^{\varrho_{1} ; \xi} \omega(\vartheta)=\omega(\vartheta)
$$

Lemma 2.4 $([1,9])$ Let $\varrho_{1}, \varrho_{2}>0$ and $\delta>0$. If $\psi(\vartheta):=(\xi(\vartheta)-\xi(a))^{\delta-1}$, then

(1) $\mathcal{I}_{a+}^{\varrho_{1} ; \xi} \psi(\vartheta)=\frac{\Gamma(\delta)}{\Gamma\left(\varrho_{1}+\delta\right)}(\xi(\vartheta)-\xi(a))^{\varrho_{1}+\delta-1}$;

(2) $\mathcal{D}_{a+}^{\varrho_{1}, \varrho_{2} ; \xi} \psi(\vartheta)=\frac{\Gamma(\delta)}{\Gamma\left(\delta-\varrho_{1}\right)}(\xi(\vartheta)-\xi(a))^{\varrho_{1}-\delta-1}, \delta>\gamma=\varrho_{1}+\varrho_{2}\left(n-\varrho_{1}\right)$;

(3) $\mathcal{D}_{a+}^{\varrho_{1}, \varrho_{2} ; \xi} \psi(\vartheta)=0$ for $\delta=\gamma$.

Lemma 2.5 ([9]) Let $\varrho_{1} \in(n-1, n](n \in \mathbb{N}), \varrho_{2} \in[0,1]$ with $\gamma=\varrho_{1}+\varrho_{2}\left(n-\varrho_{1}\right)$ and $\omega \in \mathcal{C}^{n}$. Then

$$
\left(\mathcal{I}_{a^{+}}^{\varrho_{1} ; \xi} \mathcal{D}_{a^{+}}^{\varrho_{1}, \varrho_{2} ; \xi} \omega\right)(\vartheta)=\omega(\vartheta)-\sum_{k=1}^{n} \frac{(\xi(\vartheta)-\xi(a))^{\gamma-k}}{\Gamma(\gamma-k+1)} D_{\xi}^{n}\left(\mathcal{I}_{a^{+}}^{\left(1-\varrho_{2}\right)\left(n-\varrho_{1}\right) ; \xi} \omega\right)(a) .
$$

In particular, if $1<\varrho_{1} \leq 2,0 \leq \varrho_{2} \leq 1$,where $\gamma=\varrho_{1}+\varrho_{2}\left(2-\varrho_{1}\right)$, then

$$
\begin{aligned}
\left(\mathcal{I}_{a^{+}}^{\varrho_{1} ; \xi} \mathcal{D}_{a^{+}}^{\varrho_{1}, \varrho_{2} ; \xi} \omega\right)(\vartheta)= & \omega(\vartheta)-\frac{(\xi(\vartheta)-\xi(a))^{\gamma-1}}{\Gamma(\gamma)}\left(\mathcal{I}_{a^{+}}^{1-\gamma ; \xi} \omega\right)(a) \\
& -\frac{(\xi(\vartheta)-\xi(a))^{\gamma-2}}{\Gamma(\gamma-1)}\left(\mathcal{I}_{a^{+}}^{2-\gamma ; \xi} \omega\right)(a)
\end{aligned}
$$

Lemma 2.6 ([1]) Let $n-1 \leq \varrho_{1}<n$ and $\omega \in \mathcal{C}$. Then

$$
\left(\mathcal{I}_{a+}^{\varrho_{1+} ; \xi} \omega\right)(a)=\lim _{\vartheta \rightarrow a_{+}}\left(\mathcal{I}_{a+}^{\varrho_{1} ; \xi} \omega\right)(\vartheta)=0
$$

Lemma 2.7 Let $\varrho_{1}, \theta>0$. Then, for all $\vartheta \in \mathscr{J}$, we have

$$
\mathcal{I}_{a^{+}}^{\varrho_{1} ; \xi} e^{\theta(\xi(\vartheta)-\xi(a))} \leq \frac{e^{\theta(\xi(\vartheta)-\xi(a))}}{\theta^{\varrho_{1}}} .
$$

Proof From equation (2.3), we have

$$
\mathcal{I}_{a^{+}}^{\varrho_{1} ; \phi} e^{\theta(\xi(\vartheta)-\xi(a))}=\frac{1}{\Gamma\left(\varrho_{1}\right)} \int_{a}^{\vartheta} \xi^{\prime}(s)(\xi(\vartheta)-\xi(s))^{\varrho_{1}-1} e^{\theta(\xi(s)-\xi(a))} \mathrm{d} s .
$$

By utilizing the change of variables $y=\xi(\vartheta)-\xi(s)$, we obtain

$$
\mathcal{I}_{a^{+}}^{\varrho_{1}, \xi} e^{\theta(\xi(\vartheta)-\xi(a))}=\frac{e^{\theta(\xi(\vartheta)-\xi(a))}}{\Gamma\left(\varrho_{1}\right)} \int_{0}^{\xi(\vartheta)-\xi(a)} \mathrm{y}^{\varrho_{1}-1} e^{-\theta y} \mathrm{dy} .
$$


Again, by utilizing the change of variables $v=\theta y$ in (2.6), we attain

$$
\begin{aligned}
\mathcal{I}_{a^{+}}^{\varrho_{1}, \xi} e^{\theta(\xi(\vartheta)-\xi(a))} & =\frac{e^{\theta(\xi(\vartheta)-\xi(a))}}{\Gamma\left(\varrho_{1}\right) \theta^{\varrho_{1}}} \int_{0}^{\theta(\xi(\vartheta)-\xi(a))} \mathrm{v}^{\varrho_{1}-1} e^{-\mathrm{v}} \mathrm{dv} \\
& \leq \frac{e^{\theta(\xi(\vartheta)-\xi(a))}}{\Gamma\left(\varrho_{1}\right) \theta^{\varrho}} \int_{0}^{\infty} \mathrm{v}^{\varrho_{1}-1} e^{-\mathrm{v}} \mathrm{dv} \\
& =\frac{e^{\theta(\xi(\vartheta)-\xi(a))}}{\theta^{\varrho_{1}}}
\end{aligned}
$$

This completes the proof.

Let us now conclude this section by recalling the following fixed point theorems.

Theorem 2.8 (Banach [36]) Let $\mathcal{E}$ be a nonempty closed subset of a Banach space X and $\mathcal{H}: \mathcal{E} \rightarrow \mathcal{E}$ be a strict contraction mapping, i.e., $\|\mathcal{H} z-\mathcal{H} y\| \leq k\|z-y\|$ for all $z, y \in \mathcal{E}$ and for some $k \in(0,1)$. Then $\mathcal{H}$ possesses a unique fixed point.

Theorem 2.9 (Kransnoselskii [37]) Let X be a Banach space. Let $\mathcal{S}$ be a nonempty convex, closed, and bounded subset of $\mathrm{X}$, and let $\mathcal{H}_{1}, \mathcal{H}_{2}$ be mappings from $\mathcal{S}$ to $\mathrm{X}$ such that:

(i) $\mathcal{H}_{1} z+\mathcal{H}_{2} y \in \mathcal{S}$ whenever $z, y \in \mathcal{S}$;

(ii) $\mathcal{H}_{1}$ is continuous and compact;

(iii) $\mathcal{H}_{2}$ is a strict contraction. Then there exists $z \in \mathcal{S}$ such that $z=\mathcal{H}_{1} z+\mathcal{H}_{2} z$.

\section{Main result}

The next lemma transacts with a linear form associated with problem (1.1)-(1.2).

Lemma 3.1 Let $1<\varrho_{1} \leq 2,0 \leq \varrho_{2} \leq 1$, where $\gamma=\varrho_{1}+\varrho_{2}\left(2-\varrho_{1}\right)$ and $h \in \mathcal{C}$. If

$$
A=\left[\frac{[\xi(b)-\xi(a)]^{\gamma-2}}{\Gamma(\gamma-1)}-\sum_{i=1}^{m-2} \lambda_{i} \frac{\left[\xi\left(\varkappa_{i}\right)-\xi(a)\right]^{\gamma-2}}{\Gamma(\gamma-1)}\right] \neq 0,
$$

then the function $z \in \mathcal{C}$ is a solution of the linear-type problem

$$
\left\{\begin{array}{l}
\mathcal{D}_{a^{+}}^{\varrho_{1}, \varrho_{2} ; \xi} z(\vartheta)=h(\vartheta), \quad \varrho_{1} \in(1,2), \vartheta \in \mathscr{J}:=[a, b], \\
z(a)=z_{a}+\mathfrak{p}(z), \quad z_{\xi}^{\prime}(b)=z_{b}+\sum_{i=1}^{m-2} \lambda_{i} z_{\xi}^{\prime}\left(\varkappa_{i}\right)
\end{array}\right.
$$

if and only if

$$
\begin{aligned}
z(\vartheta)= & \mathcal{I}_{a^{+}}^{\varrho_{1} \xi \xi} h(\vartheta)+\frac{[\xi(\vartheta)-\xi(a)]^{\gamma-1}}{A \Gamma(\gamma)}\left[z_{b}+\mathcal{I}_{a^{+}}^{\varrho_{1}-1 ; \xi} h(b)+\sum_{i=1}^{m-2} \lambda_{i} \mathcal{I}_{a^{+}}^{\varrho_{1}-1 ; \xi} h\left(\varkappa_{i}\right)\right] \\
& +\frac{\left[z_{a}+\mathfrak{p}(z)\right]}{A}\left[\frac{[\xi(b)-\xi(a)]^{\gamma-3}+\sum_{i=1}^{m-2} \lambda_{i}\left[\xi\left(\varkappa_{i}\right)-\xi(a)\right]^{\gamma-3}}{\Gamma(\gamma-2)}+\frac{[\xi(\vartheta)-\xi(a)]^{\gamma-2}}{\Gamma(\gamma-1)}\right] .
\end{aligned}
$$

Proof The first equation of (3.2) can be written as

$$
\mathcal{I}_{a^{+}}^{2-\varrho_{1} ; \xi} \mathcal{D}_{a^{+}}^{2 ; \xi} \mathcal{I}_{a^{+}}^{\left(1-\varrho_{2}\right)\left(2-\varrho_{1}\right) ; \xi} z(\vartheta)=h(\vartheta) .
$$


Applying the operator $\mathcal{I}_{a^{+}}^{\varrho_{1} ; \xi}$, we get

$$
\mathcal{I}_{a^{+}}^{\varrho_{1} ; \xi} \mathcal{I}_{a^{+}}^{2-\varrho_{1} ; \xi} \mathcal{D}_{a^{+}}^{2 ; \xi} \mathcal{I}_{a^{+}}^{\left(1-\varrho_{2}\right)\left(2-\varrho_{1}\right) ; \xi} z(\vartheta)=\mathcal{I}_{a^{+}}^{\varrho_{1} ; \xi} h(\vartheta)
$$

which implies

$$
\mathcal{I}_{a^{+}}^{\gamma ; \xi L} \mathcal{D}_{a^{+}}^{\gamma ; \xi} z(\vartheta)=\mathcal{I}_{a^{+}}^{\varrho_{1} ; \xi} h(\vartheta), \quad \gamma=\varrho_{1}+\varrho_{2}\left(2-\varrho_{1}\right)
$$

By Lemma 2.5 and setting $\mathcal{I}_{a^{+}}^{2-\gamma ; \xi} z(a)=c_{1}, \mathcal{I}_{a^{+}}^{1-\gamma ; \xi} z(a)=c_{2}$, we have

$$
z(\vartheta)=\frac{c_{2}}{\Gamma(\gamma)}[\xi(\vartheta)-\xi(a)]^{\gamma-1}+\frac{c_{1}}{\Gamma(\gamma-1)}[\xi(\vartheta)-\xi(a)]^{\gamma-2}+\mathcal{I}_{a^{+}}^{\varrho_{1} ; \xi} h(\vartheta)
$$

Differentiation of (3.4) with the fact that $\mathcal{D}_{\xi}^{k} \mathcal{I}_{a^{+}}^{\sigma, \xi}=\mathcal{I}_{a^{+}}^{\sigma-k, \xi}$ for $k=0,1, \ldots, n-1, \sigma>k$ (see [12]) leads to

$$
z_{\xi}^{\prime}(\vartheta)=\frac{z^{\prime}(\vartheta)}{\xi^{\prime}(\vartheta)}=\frac{c_{2}(\gamma-1)}{\Gamma(\gamma)}[\xi(\vartheta)-\xi(a)]^{\gamma-2}+\frac{c_{1}(\gamma-2)}{\Gamma(\gamma-1)}[\xi(\vartheta)-\xi(a)]^{\gamma-3}+\mathcal{I}_{a^{+}}^{\varrho_{1}-1 ; \xi} h(\vartheta) .
$$

From the boundary conditions of (3.2), we obtain $c_{1}=z_{a}+\mathfrak{p}(z)$ and

$$
\begin{aligned}
& \frac{c_{2}}{\Gamma(\gamma-1)}[\xi(b)-\xi(a)]^{\gamma-2}+\frac{\left[z_{a}+\mathfrak{p}(z)\right]}{\Gamma(\gamma-2)}[\xi(b)-\xi(a)]^{\gamma-3}+\mathcal{I}_{a^{+}}^{\varrho_{1}-1 ; \xi} h(b) \\
& =z_{b}+\sum_{i=1}^{m-2} \lambda_{i}\left[\frac{c_{2}}{\Gamma(\gamma-1)}\left[\xi\left(\varkappa_{i}\right)-\xi(a)\right]^{\gamma-2}+\frac{\left[z_{a}+\mathfrak{p}(z)\right]}{\Gamma(\gamma-2)}\left[\xi\left(\varkappa_{i}\right)-\xi(a)\right]^{\gamma-3}\right. \\
& \left.\quad+\mathcal{I}_{a^{+}}^{\varrho_{1}-1 ; \xi} h\left(\varkappa_{i}\right)\right] .
\end{aligned}
$$

It follows that

$$
\begin{gathered}
c_{2}\left[\frac{[\xi(b)-\xi(a)]^{\gamma-2}}{\Gamma(\gamma-1)}-\sum_{i=1}^{m-2} \lambda_{i} \frac{\left[\xi\left(\varkappa_{i}\right)-\xi(a)\right]^{\gamma-2}}{\Gamma(\gamma-1)}\right] \\
=\frac{\left[z_{a}+\mathfrak{p}(z)\right]}{\Gamma(\gamma-2)}\left[\sum_{i=1}^{m-2} \lambda_{i}\left[\xi\left(\varkappa_{i}\right)-\xi(a)\right]^{\gamma-3}-[\xi(b)-\xi(a)]^{\gamma-3}\right] \\
+z_{b}+\sum_{i=1}^{m-2} \lambda_{i} \mathcal{I}_{a^{+}}^{\varrho \alpha_{1}-1 ; \xi} h\left(\varkappa_{i}\right)-\mathcal{I}_{a^{+}}^{\varrho_{1}-1 ; \xi} h(b) .
\end{gathered}
$$

Therefore,

$$
\begin{aligned}
c_{2}= & \frac{1}{A}\left[z_{b}+\sum_{i=1}^{m-2} \lambda_{i} \mathcal{I}_{a^{+}}^{\varrho_{1}-1 ; \xi} h\left(\varkappa_{i}\right)-\mathcal{I}_{a^{+}}^{\varrho_{1}-1 ; \xi} h(b)\right. \\
& \left.+\frac{\left[z_{a}+\mathfrak{p}(z)\right]}{\Gamma(\gamma-2)}\left(\sum_{i=1}^{m-2} \lambda_{i}\left[\xi\left(\varkappa_{i}\right)-\xi(a)\right]^{\gamma-3}-[\xi(b)-\xi(a)]^{\gamma-3}\right)\right] .
\end{aligned}
$$


Substituting the values of $c_{1}, c_{2}$ into (3.4), we get

$$
\begin{aligned}
z(\vartheta)= & \mathcal{I}_{a^{+}}^{\varrho_{1} ; \xi} h(\vartheta)+\frac{[\xi(\vartheta)-\xi(a)]^{\gamma-1}}{A \Gamma(\gamma)}\left[z_{b}+\sum_{i=1}^{m-2} \lambda_{i} \mathcal{I}_{a^{+}}^{\varrho_{1}-1 ; \xi} h\left(\varkappa_{i}\right)-\mathcal{I}_{a^{+}}^{\varrho_{1}-1 ; \xi} h(b)\right] \\
& +\frac{\left[z_{a}+\mathfrak{p}(z)\right]}{A}\left[\frac{\left(\sum_{i=1}^{m-2} \lambda_{i}\left[\xi\left(\varkappa_{i}\right)-\xi(a)\right]^{\gamma-3}-[\xi(b)-\xi(a)]^{\gamma-3}\right)}{\Gamma(\gamma-2)}\right. \\
& \left.+\frac{[\xi(\vartheta)-\xi(a)]^{\gamma-2}}{\Gamma(\gamma-1)}\right] .
\end{aligned}
$$

Note that

$$
I_{a^{+}}^{\theta ; \xi} h(\vartheta)=\frac{1}{\Gamma(\theta)} \int_{a}^{x} \xi^{\prime}(s)[\xi(\vartheta)-\xi(s)]^{\theta-1} h(s) \mathrm{d} s, \quad \theta \in\left\{\varrho_{1}, \varrho_{1}-1\right\}, \text { and } x \in\left\{\vartheta, b, \varkappa_{i}\right\} .
$$

The converse follows by direct calculation with the aid of the results in Lemmas 2.4, 2.5. This finishes the proof.

To follow up, we need the following assumptions.

(G1) $\mathcal{F}: \mathscr{J} \times \mathscr{R} \rightarrow \mathscr{R}$ and $\mathfrak{p}: \mathcal{C} \rightarrow \mathscr{R}$ are continuous.

(G2) There exist constants $\mathcal{L}_{1}, \mathcal{L}_{2}>0$ such that

$$
|\mathcal{F}(\vartheta, z)-\mathcal{F}(\vartheta, \bar{z})| \leq \mathcal{L}_{1}|z-\bar{z}|, \quad z, \bar{z} \in \mathbb{R}
$$

and

$$
|\mathfrak{p}(z)-\mathfrak{p}(\bar{z})| \leq \mathcal{L}_{2}|z-\bar{z}|, \quad z, \bar{z} \in \mathcal{C}
$$

(G3) There exist positive functions $\varphi, \phi$ with bounds $\|\varphi\|$ and $\|\phi\|$, respectively, such that:

$$
\begin{aligned}
& |\mathcal{F}(\vartheta, z)| \leq \varphi(\vartheta), \quad \forall(\vartheta, z) \in \mathscr{J} \times \mathbb{R}, \\
& |\mathfrak{p}(z)| \leq \phi(\vartheta), \quad \forall(\vartheta, z) \in \mathscr{J} \times \mathcal{C} .
\end{aligned}
$$

For simplicity, we denote

$$
\begin{aligned}
\mathcal{M}:= & \sup _{\vartheta \in[a, b]}|\mathcal{F}(\vartheta, 0)|, \quad|\mathfrak{p}(0)|:=\mathfrak{p}_{0}, \\
\Delta:= & {\left[\mathcal { L } _ { 1 } \left(\frac{(\xi(b)-\xi(a))^{\varrho_{1}}}{\Gamma\left(\varrho_{1}+1\right)}+\frac{[\xi(b)-\xi(a)]^{\gamma-1}}{A \Gamma(\gamma)}\right.\right.} \\
& \left.\times\left[\frac{(\xi(b)-\xi(a))^{\varrho_{1}-1}}{\Gamma\left(\varrho_{1}\right)}+\sum_{i=1}^{m-2} \lambda_{i} \frac{\left(\xi\left(\varkappa_{i}\right)-\xi(a)\right)^{\varrho_{1}-1}}{\Gamma\left(\varrho_{1}\right)}\right]\right) \\
& \left.+\mathcal{L}_{2}\left[\frac{[\xi(b)-\xi(a)]^{\gamma-3}+\sum_{i=1}^{m-2} \lambda_{i}\left[\xi\left(\varkappa_{i}\right)-\xi(a)\right]^{\gamma-3}}{A \Gamma(\gamma-2)}+\frac{[\xi(b)-\xi(a)]^{\gamma-2}}{A \Gamma(\gamma-1)}\right]\right] \\
\Theta:= & {\left[\mathcal{L}_{1}\left(\frac{[\xi(b)-\xi(a)]^{\gamma-1}}{A \Gamma(\gamma)}\left[\frac{\left(\xi(b)-\xi(a) \varrho^{\varrho_{1}-1}\right.}{\Gamma\left(\varrho_{1}\right)}+\sum_{i=1}^{m-2} \lambda_{i} \frac{\left(\xi\left(\varkappa_{i}\right)-\xi(a)\right)^{\varrho_{1}-1}}{\Gamma\left(\varrho_{1}\right)}\right]\right)\right.}
\end{aligned}
$$




$$
\begin{aligned}
& \left.+\mathcal{L}_{2}\left[\frac{[\xi(b)-\xi(a)]^{\gamma-3}+\sum_{i=1}^{m-2} \lambda_{i}\left[\xi\left(\varkappa_{i}\right)-\xi(a)\right]^{\gamma-3}}{A \Gamma(\gamma-2)}+\frac{[\xi(b)-\xi(a)]^{\gamma-2}}{A \Gamma(\gamma-1)}\right]\right], \\
& \mu(\vartheta):=\frac{[\xi(\vartheta)-\xi(a)]^{\gamma-1}}{A \Gamma(\gamma)}, \\
& \nu(\vartheta):=\left[\frac{[\xi(b)-\xi(a)]^{\gamma-3}+\sum_{i=1}^{m-2} \lambda_{i}\left[\xi\left(\varkappa_{i}\right)-\xi(a)\right]^{\gamma-3}}{A \Gamma(\gamma-2)}+\frac{[\xi(\vartheta)-\xi(a)]^{\gamma-2}}{A \Gamma(\gamma-1)}\right], \\
& \Lambda_{1}:=\left[\frac{(\xi(b)-\xi(a))^{\varrho_{1}}}{\Gamma\left(\varrho_{1}+1\right)}+\mu^{*}\left(\frac{(\xi(b)-\xi(a))^{\varrho_{1}-1}}{\Gamma\left(\varrho_{1}\right)}+\sum_{i=1}^{m-2} \lambda_{i} \frac{\left(\xi\left(\varkappa_{i}\right)-\xi(a)\right)^{\varrho_{1}-1}}{\Gamma\left(\varrho_{1}\right)}\right)\right], \\
& \Lambda_{2}:=v^{*}, \quad \Lambda_{3}:=\mu^{*}\left|z_{b}\right|+v^{*}\left|z_{a}\right|, \\
& \mathcal{G}_{\xi}^{\chi}(\vartheta, s)=\frac{\xi^{\prime}(s)(\xi(\vartheta)-\xi(s))^{\chi-1}}{\Gamma(\chi)}, \quad \chi>0, \\
& \mu^{*}=\max _{0 \leq \vartheta \leq b}|\mu(\vartheta)|, \\
& v^{*}=\max _{0<\vartheta<b}|v(\vartheta)| .
\end{aligned}
$$

In what follows, we present the needful lemma that represents the equivalent solution to problem (1.1)-(1.2).

Lemma 3.2 Assume that $\mathcal{F}: \mathscr{J} \times \mathscr{R} \rightarrow \mathscr{R}, \mathfrak{p}: \mathcal{C} \rightarrow \mathscr{R}$ are continuous. A function $z(\vartheta)$ solves system (1.1)-(1.2) if and only if it is a fixed point of the operator $\mathcal{H}: \mathcal{C} \rightarrow \mathcal{C}$ defined by

$$
\begin{aligned}
\mathcal{H} z(\vartheta)= & \mathcal{I}_{a^{+}}^{\varrho_{1} ; \xi} \mathcal{F}(\vartheta, z(\vartheta))+\mu(\vartheta)\left[z_{b}+\mathcal{I}_{a^{+}}^{\varrho_{1}-1 ; \xi} \mathcal{F}(b, z(b))+\sum_{i=1}^{m-2} \lambda_{i} \mathcal{I}_{a^{+}}^{\varrho_{1}-1 ; \xi} \mathcal{F}\left(\varkappa_{i}, z\left(\varkappa_{i}\right)\right)\right] \\
& +v(\vartheta)\left[z_{a}+\mathfrak{p}(z)\right] .
\end{aligned}
$$

\subsection{Uniqueness results via Theorem 2.8}

Theorem 3.3 Assume that (G1)-(G2) hold. If

$$
\Delta:=\mathcal{L}_{1} \Lambda_{1}+\mathcal{L}_{2} \Lambda_{2}<1
$$

then there exists a unique solution for (1.1)-(1.2) on $\mathscr{J}$, where $\Delta$ is given by (3.6).

Proof According to Lemma 3.1, we consider the operator $\mathcal{H}: \mathcal{C} \rightarrow \mathcal{C}$ defined by (3.12). Then we prove that $\mathcal{H}$ has a fixed point due to Theorem 2.8. First, we define the closed ball $\mathcal{B}_{\mathcal{R}}=\{z \in \mathcal{C}:\|z\| \leq \mathcal{R}\} \subset \mathcal{C}$ with $\mathcal{R}>\frac{\mathcal{M} \Lambda_{1}+\Lambda_{3}}{1-\left(\mathcal{L}_{1} \Lambda_{1}+\mathcal{L}_{2} \Lambda_{2}\right)}$. Then, for $z \in \mathcal{B}_{\mathcal{R}}$, we have

$$
\begin{aligned}
\mid \mathcal{F}(s, z(s) \mid & =|\mathcal{F}(s, z(s))-\mathcal{F}(s, 0)+\mathcal{F}(s, 0)| \\
& \leq|\mathcal{F}(s, z(s))-\mathcal{F}(s, 0)|+|\mathcal{F}(s, 0)| \\
& \leq\left(\mathcal{L}_{1}|z(s)|+|\mathcal{F}(s, 0)|\right) \\
& \leq \mathcal{L}_{1} \mathcal{R}+\mathcal{M} .
\end{aligned}
$$


Similarly, we obtain

$$
|\mathfrak{p}(z)| \leq \mathcal{L}_{2} \mathcal{R}
$$

Hence

$$
\left.\begin{array}{rl}
|\mathcal{H} z(\vartheta)|= & v(\vartheta)\left[\left|z_{a}\right|+|\mathfrak{p}(z)|\right]+\int_{a}^{\vartheta} \mathcal{G}_{\xi}^{\varrho_{1}}(\vartheta, s)|\mathcal{F}(s, z(s))| \mathrm{d} \mathbf{s} \\
& +\mu(\vartheta)\left[\left|z_{b}\right|+\int_{a}^{b} \mathcal{G}_{\xi}^{\varrho_{1}-1}(b, s)|\mathcal{F}(s, z(s))| \mathrm{d} \mathbf{s}\right. \\
& \left.+\sum_{i=1}^{m-2} \lambda_{i} \int_{a}^{\varkappa_{i}} \mathcal{G}_{\xi}^{\varrho_{1}-1}\left(\varkappa_{i}, s\right)|\mathcal{F}(s, z(s))| \mathrm{d} \mathbf{s}\right] \\
\leq & v(\vartheta)\left[\left|z_{a}\right|+\mathcal{L}_{2} \mathcal{R}\right]+\left(\mathcal{L}_{1} \mathcal{R}+\mathcal{M}\right) \int_{a}^{\vartheta} \mathcal{G}_{\xi}^{\varrho_{1}}(\vartheta, s) \mathrm{d} \mathbf{s} \\
& +\mu(\vartheta)\left[\left|z_{b}\right|+\left(\mathcal{L}_{1} \mathcal{R}+\mathcal{M}\right)\left(\int_{a}^{b} \mathcal{G}_{\xi}^{\varrho_{1}-1}(b, s) \mathrm{d} \mathbf{s}+\sum_{i=1}^{m-2} \lambda_{i} \int_{a}^{\varkappa_{i}} \mathcal{G}_{\xi}^{\varrho_{1}-1}\left(\varkappa_{i}, s\right) \mathrm{d} \mathbf{s}\right)\right] \\
\leq & v^{*}\left[\left|z_{a}\right|+\mathcal{L}_{2} \mathcal{R}\right]+\left(\mathcal{L}_{1} \mathcal{R}+\mathcal{M}\right) \frac{(\xi(b)-\xi(a))^{\varrho_{1}}}{\Gamma\left(\varrho_{1}+1\right)} \\
& +\mu^{*}\left[\left|z_{b}\right|+\left(\mathcal{L}_{1} \mathcal{R}+\mathcal{M}\right)\left(\frac{(\xi(b)-\xi(a))^{\varrho_{1}-1}}{\Gamma\left(\varrho_{1}\right)}+\sum_{i=1}^{m-2} \lambda_{i} \frac{\left(\xi\left(\varkappa_{i}\right)-\xi(a)\right)^{\varrho_{1}-1}}{\Gamma\left(\varrho_{1}\right)}\right)\right] \\
\leq & \left(\mathcal{L}_{1} \mathcal{R}+\mathcal{M}\right)\left[\frac{(\xi(b)-\xi(a))^{\varrho_{1}}}{\Gamma\left(\varrho_{1}+1\right)}\right. \\
& \left.+\mu^{*}\left(\frac{(\xi(b)-\xi(a))^{\varrho_{1}-1}}{\Gamma\left(\varrho_{1}\right)}+\sum_{i=1}^{m-2} \lambda_{i} \frac{\left(\xi\left(\varkappa_{i}\right)-\xi(a)\right)^{\varrho_{1}-1}}{\Gamma\left(\varrho_{1}\right)}\right)\right] \\
& +v^{*}\left[\mathcal{L}_{2} \mathcal{R}\right]+\mu^{*}\left|z_{b}\right|+v^{*}\left|z_{a}\right| \\
\leq & \left(\mathcal{L}_{1} \mathcal{R}+\mathcal{M}\right) \Lambda_{1}+\left(\mathcal{L}_{2} \mathcal{R}\right) \Lambda_{2}+\Lambda_{3} \leq \mathcal{R},
\end{array}\right]
$$

which implies that $\|\mathcal{H} z\| \leq \mathcal{R}$, i.e., $\mathcal{H} \mathcal{B}_{\mathcal{R}} \subseteq \mathcal{B}_{\mathcal{R}}$.

Now, we show that $\mathcal{H}$ is a contraction. Let $z, y \in \mathcal{C}$. Then, for every $\vartheta \in \mathscr{J}$,

$$
\begin{aligned}
|(\mathcal{H} y)(\vartheta)-(\mathcal{H} z)(\vartheta)|= & v(\vartheta)[|\mathfrak{p}(y)-\mathfrak{p}(z)|]+\int_{a}^{\vartheta} \mathcal{G}_{\xi}^{\varrho_{1}}(\vartheta, s)|\mathcal{F}(s, y(s))-\mathcal{F}(s, z(s))| \mathrm{d} \mathbf{s} \\
& +\mu(\vartheta)\left[\int_{a}^{b} \mathcal{G}_{\xi}^{\varrho_{1}-1}(b, s)|\mathcal{F}(s, y(s))-\mathcal{F}(s, z(s))| \mathrm{d} \mathbf{s}\right. \\
& \left.+\sum_{i=1}^{m-2} \lambda_{i} \int_{a}^{\varkappa_{i}} \mathcal{G}_{\xi}^{\varrho_{1}-1}\left(\varkappa_{i}, s\right)|\mathcal{F}(s, y(s))-\mathcal{F}(s, z(s))| \mathrm{d} \mathbf{s}\right] \\
\leq & v(\vartheta)\left[\mathcal{L}_{2}|y(s)-z(s)|\right]+\int_{a}^{\vartheta} \mathcal{G}_{\xi}^{\varrho_{1}}(\vartheta, s) \mathcal{L}_{1}|y(s)-z(s)| \mathrm{d} \mathbf{s} \\
& +\mu(\vartheta)\left[\int_{a}^{b} \mathcal{G}_{\xi}^{\varrho_{1}-1}(b, s) \mathcal{L}_{1}|y(s)-z(s)| \mathrm{d} \mathbf{s}\right.
\end{aligned}
$$




$$
\begin{aligned}
& \left.+\sum_{i=1}^{m-2} \lambda_{i} \int_{a}^{\varkappa_{i}} \mathcal{G}_{\xi}^{\varrho_{1}-1}\left(\varkappa_{i}, s\right) \mathcal{L}_{1}|y(s)-z(s)| \mathrm{d} \mathbf{s}\right] \\
\leq & \|y-z\|\left[\mathcal{L}_{2} v(\vartheta)+\mathcal{L}_{1}\left(\int_{a}^{\vartheta} \mathcal{G}_{\xi}^{\varrho_{1}}(\vartheta, s) \mathrm{d} \mathbf{s}+\mu(\vartheta)\right.\right. \\
& \left.\left.\times\left[\int_{a}^{b} \mathcal{G}_{\xi}^{\varrho_{1}-1}(b, s) \mathrm{d} \mathbf{s}+\sum_{i=1}^{m-2} \lambda_{i} \int_{a}^{\varkappa_{i}} \mathcal{G}_{\xi}^{\varrho_{1}-1}\left(\varkappa_{i}, s\right) \mathrm{d} \mathbf{s}\right]\right)\right] .
\end{aligned}
$$

Also note that

$$
\int_{a}^{\vartheta} \mathcal{G}_{\xi}^{\chi}(\vartheta, s) \mathrm{d} s \leq \frac{(\xi(b)-\xi(a))^{\chi}}{\Gamma(\chi+1)}, \quad \chi>0
$$

Using the above arguments, we get

$$
\begin{aligned}
\| \mathcal{H} y & -\mathcal{H} z \|_{\infty} \\
\leq & \|y-z\|_{\infty}\left[\mathcal{L}_{2} v^{*}+\mathcal{L}_{1}\left(\frac{(\xi(b)-\xi(a))^{\varrho_{1}}}{\Gamma\left(\varrho_{1}+1\right)}\right.\right. \\
& \left.\left.+\mu^{*}\left[\frac{(\xi(b)-\xi(a))^{\varrho_{1}-1}}{\Gamma\left(\varrho_{1}\right)}+\sum_{i=1}^{m-2} \lambda_{i} \frac{\left(\xi\left(\varkappa_{i}\right)-\xi(a)\right)^{\varrho_{1}-1}}{\Gamma\left(\varrho_{1}\right)}\right]\right)\right] \\
:= & \Delta\|y-z\|_{\infty} .
\end{aligned}
$$

As $\Delta<1$, we deduce that $\mathcal{H}$ is a contraction. Hence, Theorem 2.8 shows that BVP (1.1)(1.2) has a unique solution. This completes the proof.

Remark 3.4 We would like to point out that the strong condition $\Delta<1$ can be removed if we use the well-known Bielecki norm.

In fact, just like the discussion in Theorem 3.3, we only prove that $\mathcal{H}$ defined as before is a contraction on $\mathcal{C}$ via the Bielecki norm. Given $z, y \in \mathcal{C}$ and $\vartheta \in \mathscr{J}$, using (G2) and Lemma 2.7, we have

$$
\begin{aligned}
|(\mathcal{H} y)(\vartheta)-(\mathcal{H} z)(\vartheta)| & \\
\leq & v(\vartheta)[|\mathfrak{p}(y)-\mathfrak{p}(z)|]+\int_{a}^{\vartheta} \mathcal{G}_{\xi}^{\varrho_{1}}(\vartheta, s)|\mathcal{F}(s, y(s))-\mathcal{F}(s, z(s))| \mathrm{d} \mathbf{s} \\
& +\mu(\vartheta)\left[\int_{a}^{b} \mathcal{G}_{\xi}^{\varrho_{1}-1}(b, s)|\mathcal{F}(s, y(s))-\mathcal{F}(s, z(s))| \mathrm{d} \mathbf{s}\right. \\
& \left.+\sum_{i=1}^{m-2} \lambda_{i} \int_{a}^{\varkappa_{i}} \mathcal{G}_{\xi}^{\varrho_{1}-1}\left(\varkappa_{i}, s\right)|\mathcal{F}(s, y(s))-\mathcal{F}(s, z(s))| \mathrm{d} \mathbf{s}\right] \\
\leq & v(\vartheta)\left[\frac{\mathcal{L}_{2}|y(\vartheta)-z(\vartheta)|}{e^{\theta(\xi(\vartheta)-\xi(a))}} e^{\theta(\xi(\vartheta)-\xi(a))}\right] \\
& +\int_{a}^{\vartheta} \mathcal{G}_{\xi}^{\varrho_{1}}(\vartheta, s) \frac{\mathcal{L}_{1}|y(s)-z(s)|}{e^{\theta(\xi(s)-\xi(a))}} e^{\theta(\xi(s)-\xi(a))} \mathrm{ds}
\end{aligned}
$$




$$
\begin{aligned}
& +\mu(\vartheta)\left[\int_{a}^{b} \mathcal{G}_{\xi}^{\varrho_{1}-1}(b, s) \frac{\mathcal{L}_{1}|y(s)-z(s)|}{e^{\theta(\xi(s)-\xi(a))}} e^{\theta(\xi(s)-\xi(a))} \mathrm{ds}\right. \\
& \left.+\sum_{i=1}^{m-2} \lambda_{i} \int_{a}^{\varkappa_{i}} \mathcal{G}_{\xi}^{\varrho_{1}-1}\left(\varkappa_{i}, s\right) \frac{\mathcal{L}_{1}|y(s)-z(s)|}{e^{\theta(\xi(s)-\xi(a))}} e^{\theta(\xi(s)-\xi(a))} \mathrm{ds}\right] \\
& \leq\left[v(\vartheta) \mathcal{L}_{2}\left[e^{\theta(\xi(\vartheta)-\xi(a))}\right]+\mathcal{L}_{1} \int_{a}^{\vartheta} \mathcal{G}_{\xi}^{\varrho_{1}}(\vartheta, s) e^{\theta(\xi(s)-\xi(a))} \mathrm{ds}\right. \\
& +\mu(\vartheta) \mathcal{L}_{1}\left[\int_{a}^{b} \mathcal{G}_{\xi}^{\varrho_{1}-1}(b, s) e^{\theta(\xi(s)-\xi(a))} \mathrm{ds}\right. \\
& \left.\left.+\sum_{i=1}^{m-2} \lambda_{i} \int_{a}^{\varkappa_{i}} \mathcal{G}_{\xi}^{\varrho_{1}-1}\left(\varkappa_{i}, s\right) e^{\theta(\xi(s)-\xi(a))} \mathrm{ds}\right]\right]\|y-z\|_{\theta} \\
& \leq\left[\left(v^{*} \mathcal{L}_{2}+\frac{\mathcal{L}_{1}}{\theta^{\varrho_{1}}}\right) e^{\theta(\xi(\vartheta)-\xi(a))}\right. \\
& \left.+\frac{\mu^{*} \mathcal{L}_{1}}{\theta^{\varrho_{1}-1}} e^{\theta(\xi(b)-\xi(a))}+\sum_{i=1}^{m-2} \lambda_{i} \frac{\mathcal{L}_{1} \mu^{*}}{\theta^{\varrho_{1}-1}} e^{\theta\left(\xi\left(\varkappa_{i}\right)-\xi(a)\right)}\right]\|y-z\|_{\theta},
\end{aligned}
$$

where

$$
\|z\|_{\theta}:=\sup _{\vartheta \in \mathscr{J}} \frac{|z(\vartheta)|}{e^{\theta(\xi(\vartheta)-\xi(a))}}, \quad \theta>0,
$$

denotes the Bielecki-type norm on the Banach space $\mathcal{C}$. Thus, we obtain

$$
\|\mathcal{H} y-\mathcal{H} z\|_{\theta} \leq\left[\mathcal{L}_{2} v^{*}+\mathcal{L}_{1}\left(\frac{1}{\theta^{\varrho_{1}}}+\frac{\mu^{*}}{\theta^{\varrho^{1}-1}}+\sum_{i=1}^{m-2} \lambda_{i} \frac{\mu^{*}}{\theta^{\varrho_{1}-1}}\right)\right]\|y-z\|_{\theta} .
$$

Taking $\theta>0$ large enough such that

$$
\left[\mathcal{L}_{2} v^{*}+\mathcal{L}_{1}\left(\frac{1}{\theta^{\varrho_{1}}}+\frac{\mu^{*}}{\theta^{\varrho_{1}-1}}+\sum_{i=1}^{m-2} \lambda_{i} \frac{\mu^{*}}{\theta^{\varrho_{1}-1}}\right)\right]<1,
$$

it follows that

$$
\|\mathcal{H} y-\mathcal{H} z\|_{\theta}<\|y-z\|_{\theta} .
$$

This means that $\mathcal{H}$ is a contraction with respect to the Bielecki norm. Hence, Theorem 2.8 shows that BVP (1.1)-(1.2) has a unique solution.

\subsection{Existence result via Theorem 2.9}

Theorem 3.5 Assume that (G1)-(G3) hold. Then (1.1)-(1.2) has at least one solution on $\mathscr{J}$, provided

$\Theta<1$,

where $\Theta$ is given by (3.7). 
Proof By assumption (G3), we can fix

$$
\rho \geq\|\varphi\| \Lambda_{1}+\|\phi\| \Lambda_{2}+\Lambda_{3}
$$

where $B_{\rho}=\{z \in \mathcal{C}:\|z\| \leq \rho\}$. Let us split the operator $\mathcal{H}: \mathcal{C} \rightarrow \mathcal{C}$ defined by (3.12) as $\mathcal{H}=\mathcal{H}_{1}+\mathcal{H}_{2}$, where $\mathcal{H}_{1}$ and $\mathcal{H}_{2}$ are given by

$$
\begin{aligned}
\mathcal{H}_{1} z(\vartheta)= & \int_{a}^{\vartheta} \mathcal{G}_{\xi}^{\varrho_{1}}(\vartheta, s) \mathcal{F}(s, z(s)) \mathrm{ds}, \\
\mathcal{H}_{2} z(\vartheta)= & \mu(\vartheta)\left[z_{b}+\int_{a}^{b} \mathcal{G}_{\xi}^{\varrho_{1}}(b, s) \mathcal{F}(s, z(s)) \mathrm{d} s+\sum_{i=1}^{m-2} \lambda_{i} \int_{a}^{\varkappa_{i}} \mathcal{G}_{\xi}^{\varrho_{1}}\left(\varkappa_{i}, s\right) \mathcal{F}(s, z(s)) \mathrm{ds}\right] \\
& +v(\vartheta)\left[z_{a}+\mathfrak{p}(z)\right] .
\end{aligned}
$$

The proof will be split into numerous steps.

Step 1: $\mathcal{H}_{1}(z)+\mathcal{H}_{2}\left(z_{1}\right) \in B_{\rho}$. Indeed, for each $z, z_{1} \in B_{\rho}$ and $\vartheta \in \mathscr{J}$, then

$$
\begin{aligned}
\| \mathcal{H}_{1} z & +\mathcal{H}_{2} z_{1} \| \\
= & \sup _{\vartheta \in \mathcal{J}}\left|\left(\mathcal{H}_{1} z\right)(\vartheta)+\left(\mathcal{H}_{2} z_{1}\right)(\vartheta)\right| \\
\leq & \int_{a}^{\vartheta} \mathcal{G}_{\xi}^{\varrho_{1}}(\vartheta, s)|\mathcal{F}(s, z(s))| \mathrm{d} \mathbf{s}+\mu(\vartheta) \\
& \times\left[\left|z_{b}\right|+\int_{a}^{b} \mathcal{G}_{\xi}^{\varrho_{1}-1}(b, s)|\mathcal{F}(s, z(s))| \mathrm{d} \mathbf{s}\right. \\
& \left.+\sum_{i=1}^{m-2} \lambda_{i} \int_{a}^{\varkappa_{i}} \mathcal{G}_{\xi}^{\varrho_{1}-1}\left(\varkappa_{i}, s\right)|\mathcal{F}(s, z(s))| \mathrm{d} \mathbf{s}\right] \\
& +v(\vartheta)\left[\left|z_{a}\right|+|\mathfrak{p}(z)|\right] \\
\leq & \|\varphi\|\left[\frac{(\xi(b)-\xi(a))^{\varrho_{1}}}{\Gamma\left(\varrho_{1}+1\right)}+\mu^{*}\left(\frac{(\xi(b)-\xi(a))^{\varrho_{1}-1}}{\Gamma\left(\varrho_{1}\right)}+\sum_{i=1}^{m-2} \lambda_{i} \frac{\left(\xi\left(\varkappa_{i}\right)-\xi(a)\right)^{\varrho_{1}-1}}{\Gamma\left(\varrho_{1}\right)}\right)\right] \\
& +\|\phi\| v^{*}+\left|z_{a}\right| v^{*}+\mu^{*}\left|z_{b}\right| \\
\leq & \|\varphi\| \Lambda \Lambda_{1}+\|\phi\| \Lambda_{2}+\Lambda_{3} \leq \rho .
\end{aligned}
$$

Hence

$$
\left\|\mathcal{H}_{1} z+\mathcal{H}_{2} z_{1}\right\| \leq \rho
$$

which shows that $\mathcal{H}_{1} z+\mathcal{H}_{2} z_{1} \in B_{\rho}$.

Step 2: $\mathcal{H}_{2}$ is a contraction map on $B_{\rho}$.

Due to the contractility of $\mathcal{H}$ as in Theorem $3.3, \mathcal{H}_{2}$ is a contraction map too.

Step 3: $\mathcal{H}_{1}$ is completely continuous on $B_{\rho}$.

From the continuity of $\mathcal{F}(\cdot, z(\cdot))$ it follows that $\mathcal{H}_{1}$ is continuous. 
Since

$$
\begin{aligned}
\left\|\mathcal{H}_{1} z\right\| & =\sup _{\vartheta \in \mathscr{J}}\left|\mathcal{H}_{1} z(\vartheta)\right| \leq \int_{a}^{\vartheta} \mathcal{G}_{\xi}^{\varrho_{1}}(\vartheta, s)|\mathcal{F}(s, z(s))| \mathrm{d} \mathbf{s} \\
& \leq\|\phi\| \frac{(\xi(b)-\xi(a))^{\varrho_{1}}}{\Gamma\left(\varrho_{1}+1\right)}:=\mathfrak{p}, \quad z \in B_{\rho},
\end{aligned}
$$

we get $\left\|\mathcal{H}_{1} z\right\| \leq \mathfrak{p}$, which emphasizes that $\mathcal{H}_{1}$ uniformly bounded on $B_{\rho}$.

Finally, we prove the compactness of $\mathcal{H}_{1}$.

For $z \in B_{\rho}$ and $\vartheta \in \mathscr{J}$, we can estimate the operator derivative as follows:

$$
\begin{aligned}
\left|\left(\mathcal{H}_{1} z\right)_{\xi}^{(1)}(\vartheta)\right| & \leq \int_{a}^{\vartheta} \mathcal{G}_{\xi}^{\varrho_{1}-1}(\vartheta, s)|\mathcal{F}(s, z(s))| \mathrm{d} \mathbf{s} \\
& \leq\|\varphi\| \frac{(\xi(b)-\xi(a))^{\varrho_{1}-1}}{\Gamma\left(\varrho_{1}\right)}:=\ell,
\end{aligned}
$$

where we used the fact

$$
\mathcal{D}_{\xi}^{k} \mathcal{I}_{a^{+}}^{\varrho_{1}, \xi}=\mathcal{I}_{a^{+}}^{\varrho_{1}-k, \xi}, \quad \omega_{\xi}^{(k)}(\vartheta)=\left(\frac{1}{\xi^{\prime}(\vartheta)} \frac{d}{d \vartheta}\right)^{k} \omega(\vartheta) \quad \text { for } k=0,1, \ldots, n-1
$$

Hence, for each $\vartheta_{1}, \vartheta_{2} \in \mathscr{J}$ with $a<\vartheta_{1}<\vartheta_{2}<b$ and for $z \in B_{\rho}$, we get

$$
\left|\left(\mathcal{H}_{1} z\right)\left(\vartheta_{2}\right)-\left(\mathcal{H}_{1} z\right)\left(\vartheta_{1}\right)\right|=\int_{\vartheta_{1}}^{\vartheta_{2}}\left|\left(\mathcal{H}_{1} z\right)^{\prime}(s)\right| \mathrm{d} s \leq \ell\left(\vartheta_{2}-\vartheta_{1}\right)
$$

where $\left(\vartheta_{2}-\vartheta_{1}\right)$ tends to zero independent of $z$. So, $\mathcal{H}_{1}$ is equicontinuous. In light of the previous arguments along with the Arzela-Ascoli theorem, we derive that $\mathcal{H}_{1}$ is compact on $B_{\rho}$. Thus, the hypotheses of Theorem 2.9 hold. So there exists at least one solution of (1.1)-(1.2) on $\mathscr{J}$.

Remark 3.6 In Theorem 3.5, we can exchange the roles of the operators $\mathcal{H}_{1}$ and $\mathcal{H}_{2}$ to obtain a second result by replacing (3.14) with the following condition:

$$
\mathcal{L}_{1}\left(\frac{(\xi(b)-\xi(a))^{\varrho_{1}}}{\Gamma\left(\varrho_{1}+1\right)}\right)<1
$$

\section{UH stability analysis}

In this section, we discuss UH and generalized UH stability of (1.1)-(1.2). Let $\varepsilon>0$. We consider the following inequality:

$$
\left|\mathcal{D}_{a^{+}}^{\varrho_{1}, \varrho_{2} ; \xi} \tilde{z}(\vartheta)-\mathcal{F}(\vartheta, \tilde{z}(\vartheta))\right| \leq \varepsilon, \quad \vartheta \in \mathscr{J}
$$

Definition 4.1 FDE (1.1)-(1.2) is UH stable if there exists $c_{\mathcal{F}} \in \mathbb{R}^{+}$such that, for any $\varepsilon>0$ and for each solution $\tilde{z} \in \mathcal{C}$ of inequality (4.1), there exists a solution $z \in \mathcal{U}$ of 1.1)-(1.2) with

$$
|\tilde{z}(\vartheta)-z(\vartheta)| \leq \varepsilon c_{\mathcal{F}}, \quad \vartheta \in \mathscr{J}
$$


Definition 4.2 FDE (1.1)-(1.2) is called generalized UH stable if there exists $\varphi_{\mathcal{F}} \in$ $\mathcal{C}\left(\mathscr{R}_{+}, \mathscr{R}_{+}\right)$along with $\varphi_{\mathcal{F}}(0)=0$ if, for any $\varepsilon>0$ and for each solution $\tilde{z} \in \mathcal{C}$ of inequality (4.1), a solution $z \in \mathcal{C}$ of (1.1)-(1.2) exists uniquely for which

$$
|\tilde{z}(\vartheta)-z(\vartheta)| \leq \varphi_{\mathcal{F}}(\varepsilon), \quad \vartheta \in \mathscr{J} .
$$

Remark 4.3 A function $\tilde{z} \in \mathcal{C}$ is a solution of (4.1) iff there exists a function $v \in \mathcal{C}$ (which depends on solution $\tilde{z}$ ) such that

1. $|v(\vartheta)| \leq \varepsilon, \vartheta \in \mathscr{J}$.

2. $\mathcal{D}_{a^{+}}^{\varrho_{1}, \varrho_{2} ; \xi} \tilde{z}(\vartheta)=\mathcal{F}(\vartheta, \tilde{z}(\vartheta))+v(\vartheta), \vartheta \in \mathscr{J}$.

Theorem 4.4 Let $\Delta<1$ and hypotheses (G1) and (G2) be satisfied. Then FDE (1.1)-(1.2) is UH stable on $\mathscr{J}$ and consequently generalized UH stable.

Proof Let $\varepsilon>0$ and $\widetilde{z} \in \mathcal{C}$ satisfy inequality (4.1), and let $z \in \mathcal{C}$ be the unique solution of the following problem:

$$
\left\{\begin{array}{l}
\mathcal{D}_{a^{+}}^{\varrho_{1}, \varrho_{2} ; \xi} z(\vartheta)=\mathcal{F}(\vartheta, z(\vartheta)), \quad \vartheta \in \mathscr{J}:=[a, b], \\
z(a)=z_{a}+\mathfrak{p}(z), \quad z_{\xi}^{\prime}(b)=z_{b}+\sum_{i=1}^{m-2} \lambda_{i} z_{\xi}^{\prime}\left(\varkappa_{i}\right) .
\end{array}\right.
$$

By Lemma 3.1, we have

$$
\begin{aligned}
z(\vartheta)= & \mathcal{I}_{a^{+}}^{\varrho_{1} ; \xi} h(\vartheta)+\mu(\vartheta)\left[z_{b}+\mathcal{I}_{a^{+}}^{\varrho_{1}-1 ; \xi} h(b)+\sum_{i=1}^{m-2} \lambda_{i} \mathcal{I}_{a^{+}}^{\varrho_{1}-1 ; \xi} h\left(\varkappa_{i}\right)\right]+v(\vartheta)\left[z_{a}+\mathfrak{p}(z)\right] \\
= & \int_{a}^{\vartheta} \mathcal{G}_{\xi}^{\varrho_{1}}(\vartheta, s) \mathcal{F}(s, z(s)) \mathrm{d} s \\
& +\mu(\vartheta)\left[z_{b}+\int_{a}^{b} \mathcal{G}_{\xi}^{\varrho_{1}-1}(b, s) \mathcal{F}(s, z(s)) \mathrm{ds}+\sum_{i=1}^{m-2} \lambda_{i} \int_{a}^{\varkappa_{i}} \mathcal{G}_{\xi}^{\varrho_{1}-1}\left(\varkappa_{i}, s\right) \mathcal{F}(s, z(s)) \mathrm{ds}\right] \\
& +v(\vartheta)\left[z_{a}+\mathfrak{p}(z)\right] .
\end{aligned}
$$

Since we have assumed that $\widetilde{z}$ is a solution of (4.1), we have by Remark 4.3

$$
\left\{\begin{array}{l}
\mathcal{D}_{a^{+}}^{\varrho_{1}, \varrho_{2} ; \xi} \widetilde{z}(\vartheta)=\mathcal{F}(\vartheta, \widetilde{z}(\vartheta))+v(\vartheta), \quad \vartheta \in \mathscr{J}:=[a, b] \\
\widetilde{z}(a)=z_{a}+\mathfrak{p}(\widetilde{z}), \quad \widetilde{z}_{\xi}^{\prime}(b)=z_{b}+\sum_{i=1}^{m-2} \lambda_{i} \widetilde{z}_{\xi}^{\prime}\left(\varkappa_{i}\right) .
\end{array}\right.
$$

Again by Lemma 3.1, we have

$$
\begin{aligned}
\widetilde{z}(\vartheta)= & \int_{a}^{\vartheta} \mathcal{G}_{\xi}^{\varrho_{1}}(\vartheta, s) \mathcal{F}(s, \widetilde{z}(s)) \mathrm{ds}+\int_{a}^{\vartheta} \mathcal{G}_{\xi}^{\varrho_{1}}(\vartheta, s) v(s) \mathrm{ds} \\
& +\mu(\vartheta)\left[z_{b}+\int_{a}^{b} \mathcal{G}_{\xi}^{\varrho_{1}-1}(b, s) \mathcal{F}(s, \widetilde{z}(s)) \mathrm{d} s+\int_{a}^{b} \mathcal{G}_{\xi}^{\varrho_{1}-1}(b, s) v(s) \mathrm{ds}\right. \\
& \left.+\sum_{i=1}^{m-2} \lambda_{i} \int_{a}^{\varkappa_{i}} \mathcal{G}_{\xi}^{\varrho_{1}-1}\left(\varkappa_{i}, s\right) \mathcal{F}(s, \widetilde{z}(s)) \mathrm{ds}+\sum_{i=1}^{m-2} \lambda_{i} \int_{a}^{\varkappa_{i}} \mathcal{G}_{\xi}^{\varrho_{1}-1}\left(\varkappa_{i}, s\right) v(s) \mathrm{ds}\right]
\end{aligned}
$$




$$
+v(\vartheta)\left[z_{a}+\mathfrak{p}(z)\right]
$$

Consequently, for each $\vartheta \in \mathscr{J}$, we have

$$
\begin{aligned}
\mid \widetilde{z}(\vartheta) & -z(\vartheta) \mid \\
= & \int_{a}^{\vartheta} \mathcal{G}_{\xi}^{\varrho_{1}}(\vartheta, s)|\mathcal{F}(s, \widetilde{z}(s))-\mathcal{F}(s, z(s))| \mathrm{ds}+\int_{a}^{\vartheta} \mathcal{G}_{\xi}^{\varrho_{1}}(\vartheta, s) v(s) \mathrm{ds} \\
& +\mu(\vartheta)\left[\int_{a}^{b} \mathcal{G}_{\xi}^{\varrho_{1}-1}(b, s)|\mathcal{F}(s, \widetilde{z}(s))-\mathcal{F}(s, z(s))| \mathrm{ds}+\int_{a}^{b} \mathcal{G}_{\xi}^{\varrho_{1}-1}(b, s) v(s) \mathrm{d} s\right. \\
& +\sum_{i=1}^{m-2} \lambda_{i} \int_{a}^{\varkappa_{i}} \mathcal{G}_{\xi}^{\varrho_{1}-1}\left(\varkappa_{i}, s\right)|\mathcal{F}(s, \widetilde{z}(s))-\mathcal{F}(s, z(s))| \mathrm{ds} \\
& \left.+\sum_{i=1}^{m-2} \lambda_{i} \int_{a}^{\varkappa_{i}} \mathcal{G}_{\xi}^{\varrho_{1}-1}\left(\varkappa_{i}, s\right) v(s) \mathrm{ds}\right] \\
& +v(\vartheta)|\mathfrak{p}(\widetilde{z})-\mathfrak{p}(z)| .
\end{aligned}
$$

From Remark 4.3 and (G2), we derive

$$
\begin{aligned}
& \mid \widetilde{z}(\vartheta)-z(\vartheta) \mid \\
& \leq \Delta\|\widetilde{z}-z\| \\
& \quad+\left(\frac{(\xi(b)-\xi(a))^{\varrho_{1}}}{\Gamma\left(\varrho_{1}+1\right)}+\mu^{*}\left[\frac{(\xi(b)-\xi(a))^{\varrho_{1}-1}}{\Gamma\left(\varrho_{1}\right)}+\sum_{i=1}^{m-2} \lambda_{i} \frac{\left(\xi\left(\varkappa_{i}\right)-\xi(a)\right)^{\varrho_{1}-1}}{\Gamma\left(\varrho_{1}\right)}\right]\right) \varepsilon \\
& \quad:=\Delta\|\widetilde{z}-z\|+\Lambda \varepsilon,
\end{aligned}
$$

where $\Delta$ is defined in (3.6). In consequence, it follows that

$$
\|\widetilde{z}-z\|_{\infty} \leq \frac{\Lambda \varepsilon}{(1-\Delta)}
$$

If we set $c_{\mathcal{F}}=\frac{\Lambda}{(1-\Delta)}$, then the UH stability condition holds. Moreover, for $\varphi_{\mathcal{F}}(\varepsilon)=\frac{\Lambda \varepsilon}{(1-\Delta)}$ with $\varphi_{\mathcal{F}}(0)=0$, the generalized UH stability condition holds too. This completes the proof.

\section{Examples}

In this section, we consider two examples to better illustrate our main results.

Example 5.1 Consider the following BVP for FDE:

$$
\begin{cases}\mathcal{D}_{0^{+}}^{\frac{3}{2}, 0 ; \vartheta} z(\vartheta)=\mathcal{F}(\vartheta, z(\vartheta)), & \vartheta \in \mathscr{J}:=[0,1], \\ z(0)=\frac{1}{5}+\sum_{j=1}^{n} c_{j} z\left(\vartheta_{j}\right), & z_{\vartheta}^{\prime}(1)=\frac{2}{7}+\frac{1}{10} z^{\prime}\left(\frac{1}{6}\right)+\frac{1}{15} z^{\prime}\left(\frac{2}{9}\right),\end{cases}
$$

where

$$
\varrho_{1}=\gamma=\frac{3}{2}, \quad \varrho_{2}=0, \quad b=1, \quad a=0, \quad \xi(\vartheta)=\vartheta,
$$




$$
\begin{array}{ll}
z_{a}=\frac{1}{5} ; & z_{b}=\frac{2}{7} ; \quad \lambda_{1}=\frac{1}{10}, \quad \lambda_{2}=\frac{1}{15}, \\
\varkappa_{1}=\frac{1}{6}, \quad \varkappa_{2}=\frac{2}{9} .
\end{array}
$$

Using the given data, we find that

$$
A=\left[\frac{[b-a)]^{\gamma-2}}{\Gamma(\gamma-1)}-\sum_{i=1}^{m-2} \lambda_{i} \frac{\left[\varkappa_{i}-a\right]^{\gamma-2}}{\Gamma(\gamma-1)}\right] \simeq 0.3462 \neq 0 .
$$

In order to illustrate Theorem 3.3 and Theorem 3.5, we take

$$
\begin{aligned}
& f(\vartheta, z(\vartheta))=\frac{\vartheta^{2}-1}{\left(2-\sin ^{2} \pi \vartheta\right)^{3}}\left(\frac{|z(\vartheta)|}{1+|z(\vartheta)|}\right), \\
& \mathfrak{p}(z)=\sum_{j=1}^{n} c_{j} z\left(\vartheta_{i}\right),
\end{aligned}
$$

in (1.1), where $0<\vartheta_{1}<\vartheta_{2}<\cdots<\vartheta_{n}<c_{j}, j=1, \ldots, n$, are given positive constants with $\sum_{j=1}^{n} c_{j}<\frac{2}{5}$, and note that

$$
\begin{gathered}
\|f(\vartheta, z)-f(\vartheta, v)\| \leq \frac{1}{8}\|z-v\|, \\
\|\mathfrak{p}(z)-\mathfrak{p}(v)\|=\sum_{j=1}^{n} c_{j}\|z-v\| .
\end{gathered}
$$

Hence conditions (G1)-(G3) hold with $\mathcal{L}_{1}=\frac{1}{8}, \mathcal{L}_{2}=\frac{2}{5}$. Further, from the above given data it is easy to calculate

$$
\Theta \simeq 0.1325<1 \text {. }
$$

By Theorem 3.5, problem (5.1) with data (5.2) and (5.3) has at least a solution $z$. Furthermore $\Delta \simeq 0.2265<1$. Hence, by Theorem 3.3, problem (5.1) with data (5.2) and (5.3) has a unique solution.

Example 5.2 Let us consider BVP for FDE (1.1)-(1.2) with

$$
\begin{aligned}
& \xi(\vartheta)=2^{\vartheta}, \quad \varrho_{1}=\frac{5}{3}, \quad \varrho_{2}=\frac{1}{3}, \quad \gamma=\frac{16}{9}, \\
& z_{a}=\frac{1}{5} ; \quad z_{b}=\frac{2}{7} ; \quad \lambda_{1}=\frac{1}{5}, \quad \lambda_{2}=\frac{2}{7}, \\
& \varkappa_{1}=\frac{3}{4}, \quad \varkappa_{2}=\frac{2}{5}, \quad b=1, \quad a=0 .
\end{aligned}
$$

In order to illustrate Theorem 3.3 and Theorem 3.5, we take

$$
\begin{aligned}
& f(\vartheta, z(\vartheta))=\frac{e^{-2 \vartheta}}{\left(\vartheta^{4}+25\right)}(\sin |z(\vartheta)|), \\
& \mathfrak{p}(z)=\sum_{j=1}^{n} c_{j} z\left(\vartheta_{i}\right)
\end{aligned}
$$


in (1.1), where $0<\vartheta_{1}<\vartheta_{2}<\cdots<\vartheta_{n}<c_{j}, i=1, \ldots, n$, are given positive constants with $\sum_{j=1}^{n} c_{j}<\frac{2}{15}$, and note that

$$
\begin{aligned}
& \|f(\vartheta, z)-f(\vartheta, v)\| \leq \frac{1}{25}\|z-v\|, \\
& \|\mathfrak{p}(z)-\mathfrak{p}(v)\|=\sum_{j=1}^{n} c_{j}\|z-v\| .
\end{aligned}
$$

Hence conditions (G1)-(G3) hold with $\mathcal{L}_{1}=\frac{1}{25}$ and $\mathcal{L}_{1}=\frac{1}{15}$. Further, from the above given data, it is easy to calculate

$$
\Theta \simeq 0.7967<1 .
$$

Using the given values of the parameters in (3.1), by the Matlab program, we find that

$$
A=\left[\frac{\left[2^{b}-2^{a}\right]^{\gamma-2}}{\Gamma(\gamma-1)}-\sum_{i=1}^{m-2} \lambda_{i} \frac{\left[2^{\varkappa_{i}}-2^{a}\right]^{\gamma-2}}{\Gamma(\gamma-1)}\right] \simeq 0.3449 \neq 0 .
$$

Hence condition (G2) holds with $\mathcal{L}_{1}=\frac{1}{16}, \mathcal{L}_{2}=\frac{1}{24}$. We shall check that condition (3.13) is satisfied. Indeed, using simple calculations, we can find

$$
\Delta \simeq 0.8811<1
$$

Hence, by Theorem 3.3, BVP (1.1)-(1.2) has a unique solution on $\mathscr{J}$. Moreover, Theorem 4.4 ensures that BVP (1.1)-(1.2) is HU stable and generalized HU stable.

\section{Conclusion}

By using standard fixed point techniques, we have established some advantageous results about the existence, uniqueness, and stability of the Ulam-Hyers type of the BVP for nonlinear FDEs involving a $\xi$-Hilfer FD under nonlocal boundary conditions. The acquired results have been justified by two examples. Furthermore, problem (1.1)-(1.2) not just incorporates the formerly specified BVPs in the literature, but it does cover as many problems as special cases for various values of $\varrho_{2}$ and $\xi$, as referenced in Remarks 1.1, 1.2. Consequently, the fixed point technique is a powerful approach to investigate diverse nonlinear problems, which is very important in many theoretical and applied fields.

Acknowledgements

The authors are very thankful to the reviewers for their useful suggestions. The first and fifth authors would like to thank Prince Sultan University for funding this research work.

Funding

Prince Sultan University provided funding to support this work through research group Nonlinear Analysis Methods in Applied Mathematics (NAMAM), group number [RG-DES-2017-01-17].

Availability of data and materials

Not applicable. 
Authors' contributions

All authors contributed equally to this article. All authors read and approved the final manuscript.

\section{Author details}

${ }^{1}$ Department of Mathematics and General Sciences, Prince Sultan University, Riyadh, Saudi Arabia. ${ }^{2}$ Department of Medical Research, China Medical University Hospital, China Medical University, Taichung, 40402, Taiwan. ${ }^{3}$ Department of Mathematics, Hashemite University, Zarqa, Jordan. ${ }^{4}$ Laboratory of Mathematics And Applied Sciences, University of Ghardaia, Ghardaia, 47000, Algeria. ${ }^{5}$ Department of Mathematics, Hodeidah University, Al-Hodeidah, Yemen.

${ }^{6}$ Department of Mathematics, Imam Mohammed Ibn Saud Islamic University, Riyadh, Saudi Arabia.

\section{Publisher's Note}

Springer Nature remains neutral with regard to jurisdictional claims in published maps and institutional affiliations.

Received: 25 March 2021 Accepted: 7 June 2021 Published online: 15 June 2021

\section{References}

1. Kilbas, A.A., Srivastava, H.M., Trujillo, J.J.: Theory and Applications of Fractional Differential Equations. North-Holland Mathematics Studies, vol. 204. Elsevier, Amsterdam (2006)

2. Podlubny, I.: Fractional Differential Equations. Academic Press, New York (1999)

3. Samko, S.G., Kilbas, A.A., Marichev, O.I.: Fractional Integrals and Derivatives: Theory and Applications. Gordon \& Breach, Yverdon (1993)

4. Tarasov, V.E.: Fractional Dynamics: Application of Fractional Calculus to Dynamics of Particles, Fields and Media. Springer, Heidelberg (2010)

5. Hilfer, R.: Applications of Fractional Calculus in Physics. World Scientific, Singapore (2000)

6. Atangana, A., Baleanu, D.: New fractional derivatives with non-local and non-singular kernel: theory and application to heat transfer model. Therm. Sci. 20(2), 763-769 (2016)

7. Atangana, A.: Fractal-fractional differentiation and integration: connecting fractal calculus and fractional calculus to predict complex system. Chaos Solitons Fractals 102, 396-406 (2017)

8. Almeida, R.: A Caputo fractional derivative of a function with respect to another function. Commun. Nonlinear Sci. Numer. Simul. 44, 460-481 (2017)

9. Sousa, J.V.D.C., Oliveira, E.C.: On the $\psi$-Hilfer fractional derivative. Commun. Nonlinear Sci. Numer. Simul. 60, 72-91 (2018)

10. Agrawal, O.P.: Some generalized fractional calculus operators and their applications in integral equations. Fract. Calc. Appl. Anal. 15(4), 700-711 (2012)

11. Jarad, F., Abdeljawad, T.: Generalized fractional derivatives and Laplace transform. Discrete Contin. Dyn. Syst., Ser. S 13(3), $709(2020)$

12. Abdo, M.S., Ibrahim, A.G., Panchal, S.K.: Nonlinear implicit fractional differential equation involving $\psi$-Caputo fractional derivative. Proc. Jangjeon Math. Soc. 22(3), 387-400 (2019)

13. Abdo, M.S., Abdeljawad, T., Ali, S.M., Shah, K., Jarad, F.: Existence of positive solutions for weighted fractional order differential equations. Chaos Solitons Fractals 141, 110341 (2020)

14. Abdo, M.S.: Further results on the existence of solutions for generalized fractional quadratic functional integral equations. J. Math. Anal. Model. 1(1), 33-46 (2020)

15. Almeida, R.: Fractional differential equations with mixed boundary conditions. Bull. Malays. Math. Sci. Soc. 42(4), 1687-1697 (2019)

16. Boutiara, A., Abdo, M.S., Benbachir, M.: Existence results for $\psi$-Caputo fractional neutral functional integro-differential equations with finite delay. Turk. J. Math. 44, 2380-2401 (2020)

17. Adjimi, N., Boutiara, A., Abdo, M.S., Benbachir, M.: Existence results for nonlinear neutral generalized Caputo fractional differential equations. J. Pseudo-Differ. Oper. Appl. 12(2), 1-17 (2021)

18. Mali, A.D., Kucche, K.D.: Nonlocal boundary value problem for generalized Hilfer implicit fractional differential equations. Math. Methods Appl. Sci. 43(15), 8608-8631 (2020)

19. Sudsutad, W., Thaiprayoon, C., Ntouyas, S.K.: Existence and stability results for $\psi$-Hilfer fractional integro-differential equation with mixed nonlocal boundary conditions. AIMS Math. 6(4), 4119-4141 (2021)

20. Ntouyas, S.K., Vivek, D.: Existence and uniqueness results for sequential $\psi$-Hilfer fractional differential equations with multi-point boundary conditions. Acta Math. Univ. Comen. 90(2), 1-15 (2021)

21. Ahmed, I., Kumam, P., Abdeljawad, T., Jarad, F., Borisut, P., Demba, M.A., Kumam, W.: Existence and uniqueness results for $\varphi$-Caputo implicit fractional pantograph differential equation with generalized anti-periodic boundary condition. Adv. Differ. Equ. 2020, 555 (2020)

22. Belmor, S., Ravichandran, C., Jarad, F.: Nonlinear generalized fractional differential equations with generalized fractional integral conditions. J. Taibah Univ. Sci. 14(1), 114-123 (2020)

23. Jarad, F., Harikrishnan, S., Shah, K., Kanagarajan, K.: Existence and stability results to a class of fractional random implicit differential equations involving a generalized Hilfer fractional derivative. Discrete Contin. Dyn. Syst., Ser. S 13(3), $723(2020)$

24. Sousa, J.V.D.C., Jarad, F., Abdeljawad, T.: Existence of mild solutions to Hilfer fractional evolution equations in Banach space. Ann. Funct. Anal. 12(1), 1-16 (2021)

25. Wahash, H.A., Abdo, M.S., Saeed, A.M., Panchal, S.K.: Singular fractional differential equations with $\psi$-Caputo operator and modified Picard's iterative method. Appl. Math. E-Notes 20, 215-229 (2020)

26. Abdo, M.S., Thabet, S.T., Ahmad, B.: The existence and Ulam-Hyers stability results for $\psi$-Hilfer fractional integrodifferential equations. J. Pseudo-Differ. Oper. Appl. 11(4), 1757-1780 (2020)

27. Abdo, M.S., Panchal, S.K., Hussien, H.S.: Fractional integro-differential equations with nonlocal conditions and $\psi$-Hilfer fractional derivative. Math. Model. Anal. 24(4), 564-584 (2019) 
28. Abdo, M.S., Shah, K., Panchal, S.K., Wahash, H.A.: Existence and Ulam stability results of a coupled system for terminal value problems involving $\psi$-Hilfer fractional operator. Adv. Differ. Equ. 2020, 316 (2020)

29. Abdo, M.S., Panchal, S.K., Wahash, H.A.: Ulam-Hyers-Mittag-Leffler stability for a $\psi$-Hilfer problem with fractional order and infinite delay. Results Appl. Math. 7, 100-115 (2020)

30. Boutiara, A., Etemad, S., Hussain, A., Rezapour, S.: The generalized U-H and U-H stability and existence analysis of a coupled hybrid system of integro-differential IVPs involving $\varphi$-Caputo fractional operators. Adv. Differ. Equ. 2021, 95 (2021). https://doi.org/10.1186/s13662-021-03253-8

31. Sousa, J.V.D.C., Oliveira, E.C.: On the Ulam-Hyers-Rassias stability for nonlinear fractional differential equations using the $\psi$-Hilfer operator. J. Fixed Point Theory Appl. 20(3), 1-21 (2018)

32. Almalahi, M.A., Abdo, M.S., Panchal, S.K.: Existence and Ulam-Hyers-Mittag-Leffler stability results of $\psi$-Hilfer nonlocal Cauchy problem. Rend. Circ. Mat. Palermo 2, 1-21 (2020)

33. Zhong, W., Lin, W.: Nonlocal and multiple-point boundary value problem for fractional differential equations. Comput. Math. Appl. 59(3), 1345-1351 (2010)

34. Asawasamrit, S., Kijjathanakorn, A., Ntouyas, S.K., Tariboon, J.: Nonlocal boundary value problems for Hilfer fractional differential equations. Bull. Korean Math. Soc. 55(6), 1639-1657 (2018)

35. Srivastava, H., Tomovski, Z:: Fractional calculus with an integral operator containing a generalized Mittag-Leffler function in the kernel. Appl. Math. Comput. 211(1), 198-210 (2009)

36. Granas, A., Dugundji, J.: Fixed Point Theory. Springer, New York (2003)

37. Krasnoselskii, M.A.: Two remarks on the method of successive approximations. Usp. Mat. Nauk 10, 123-127 (1995)

\section{Submit your manuscript to a SpringerOpen ${ }^{\circ}$ journal and benefit from:}

- Convenient online submission

- Rigorous peer review

Open access: articles freely available online

- High visibility within the field

- Retaining the copyright to your article

Submit your next manuscript at $\boldsymbol{~ s p r i n g e r o p e n . c o m ~}$ 